\title{
Photoaligned liquid-crystal lens with a single low-voltage electrode
}

\author{
A.A. Muravsky ${ }^{1}$, V.E. Agabekov ${ }^{1}$, A.L. Tolstik ${ }^{2}$, U.V. Mahilny ${ }^{2}$ \\ ${ }^{1}$ Institute of Chemistry of New Materials, National Academy of Sciences of Belarus \\ 36, F. Skaryny str., 220141 Minsk, Belarus \\ Phone: +(375)17-2376788, fax: +(375)17-2376828 \\ E-mail:agabekov@ichnm.basnet.by, alexander.muravsky@ichnm.basnet.by \\ ${ }^{2}$ Belarusian State University, Physics Department \\ 4, Nezavisimosti Ave., 220030 Minsk, Belarus \\ Phone: +(375)17-2095120, Fax:+(375)17-2095445 \\ E-mail:Tolstik@bsu.by,mogilny@bsu.by
}

\begin{abstract}
The simple design of liquid-crystal lenses with single electrode and operation voltage below $5 \mathrm{~V}$ is highly desired. We offer a new type of photoaligned liquid-crystal lens, where the refractive gradient is created by voltage application due to the patterned twist angle gradient. In this paper, we present our calculations and experimental data.
\end{abstract}

Keywords: liquid-crystal lens, twist angle gradient, photoalignment.

Manuscript received 23.10.09; accepted for publication 25.03.10; published online 30.04.10.

\section{Introduction}

Simple non-mechanical lens with variable focal length is highly needed for cell-phone and web cameras. The peculiarity of the application is that it can tolerate reduction of optical quality if the benefits are lens thickness below $<2 \mathrm{~mm}$, diameter $\sim 2 \mathrm{~mm}$, driving voltage below $5 \mathrm{~V}$, low cost price and changeable focal length from $\sim 10 \mathrm{~cm}$ (i.e. short) to infinity (i.e. long) range. Today it is clear that liquid-crystal (LC) lens can perform well to satisfy these requirements.

\section{Refractive index gradient of LC lens}

LC lens requires the voltage controllable gradient of refractive index, which for the flat LC cell with fixed cell gap (Fig. 1) acts as curved surfaces of the glass lens (Fig. 2). It can be shown that for LC lens the refractive index gradient of the liquid crystal along the $y$-axis, $n_{\mathrm{LC}}(y)$ satisfies the condition:

$n_{L C}(y) d=\frac{n_{g} D}{2} \tan \left(\frac{D}{2 n_{g} f_{g}}\right)-n_{g} y \tan \left(\frac{y}{n_{g} f_{g}}\right)$,

where $d$ is the LC cell gap, $D$ - diameter of the lens, $n_{g}-$ refractive index of glass substrate material, $f_{g}$ - focal length inside the glass substrate and $F_{1}=f_{g} / n_{g}$ - focal length in the air.
At least five various approaches to LC lens design are known, which are based on patterning different LC cell components. First, it is the patterned electrode approach $[1,2]$, when multiple electrodes or gradient electrodes are created inside the LC cell to induce the gradient of electric field that controls the lens profile. The overall complexity of the device is high due to complex electronic component required. Second, it is the patterned dielectric layer [3], when the dielectric layer is applied to create the gradient of electric field controlling the lens profile. The single electrode is used, but the switching voltages are rather high. Third, it is the patterned cell gap [4], when the polymer material modifies the gap thickness of LC cell to create the curved interface, which disappears due to index matching in the ON-state. The single electrode is used, but switching voltages are high and the poor alignment of LC causes parasitic scattering. Fourth, it is the patterned LC layer [3], when the density of liquidcrystal material is modified according to monomer gradient in the bulk of LC cell. The single electrode is used, but switching voltages are also high. Fifth, it is the patterned alignment layer that affects bulk LC switching upon voltage application to the single electrode. There are several possibilities of alignment layer patterning, e.g. related to anchoring energy [5] or pretilt angle [6]. Here we present original design of liquid-crystal lens based on twist angle gradient inside LC cell with a single lowvoltage electrode. 


\section{LC lens based on twist angle gradient}

\subsection{Threshold voltage vs. twist angle}

The threshold voltage of liquid crystal switching, $V_{\text {th }}$, depends on the twist angle, $\Phi$, inside the LC cell in the following way [7]:

$V_{t h}=\pi \sqrt{\frac{K_{11}}{\varepsilon_{0} \Delta \varepsilon}} \sqrt{1+\left\{\left[\frac{K_{33}}{K_{11}}-2 \frac{K_{22}}{K_{11}}\right] \frac{\Phi}{\pi}+4 \frac{K_{22}}{K_{11}} \frac{d}{p}\right\} \frac{\Phi}{\pi}}$,

where $K_{11}, K_{22}, K_{33}$ are elastic constants of a liquid crystal, $\Delta \varepsilon-$ anisotropy of dielectric permittivity, $p-$ chiral pitch.

Fig. 3 shows the threshold voltage dependence on the twist angle for the liquid crystal E7 with $d / p=0.25$.

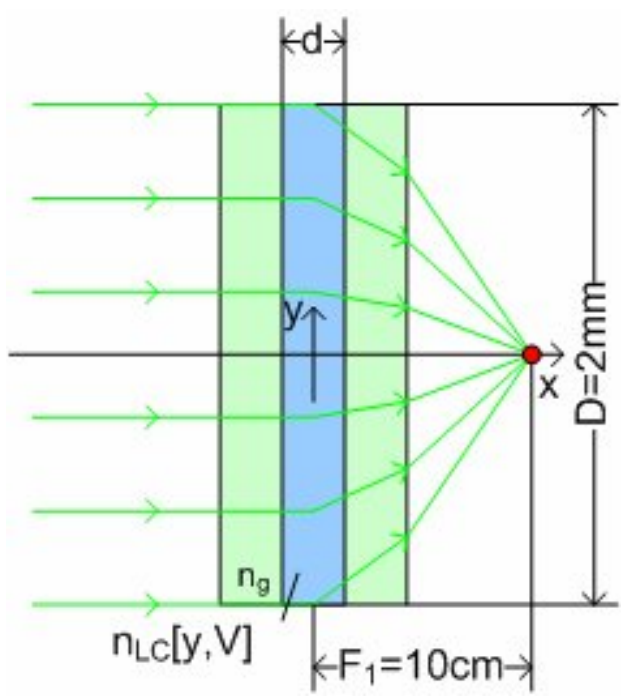

Fig. 1. Refractive index gradient of LC lens.

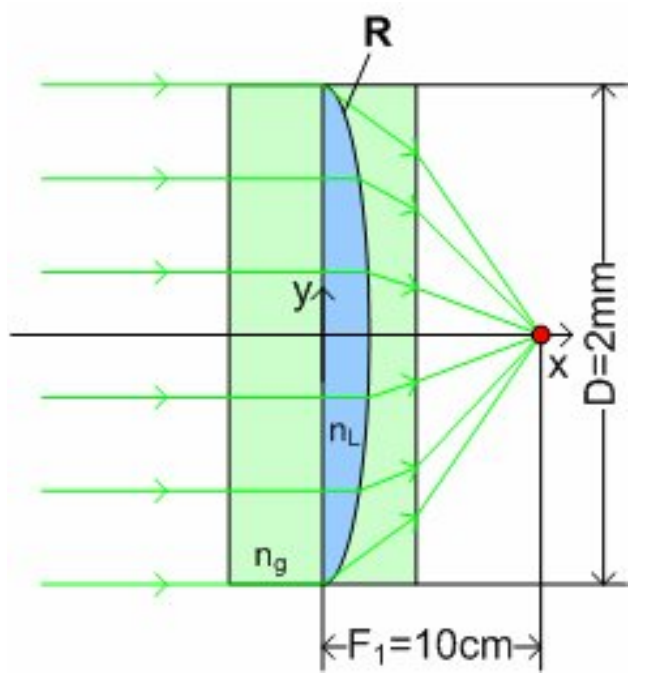

Fig. 2. Curved interface of a glass lens.

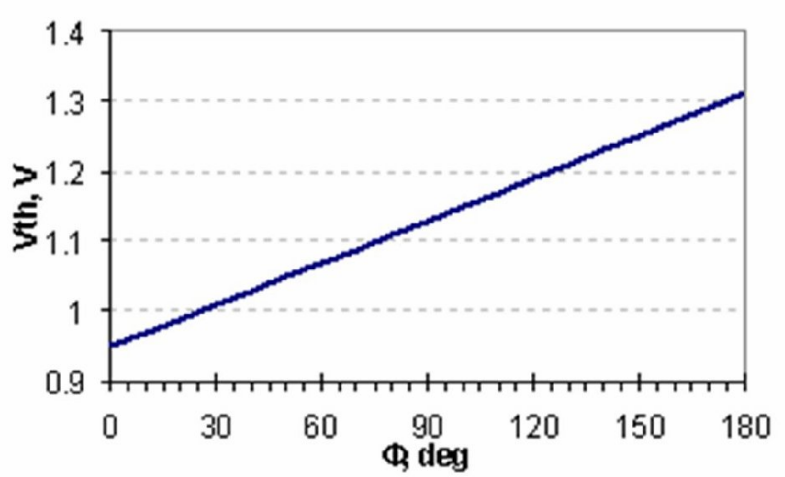

Fig. 3. Threshold voltage dependence on the twist angle for LC $\mathrm{E} 7, d / p=0.25$.

\subsection{Twist angle gradient}

Fig. 4 shows the calculated distribution of twist angle that results into phase profile for LC lens with $D=2 \mathrm{~mm}$. To avoid alignment of defects related with a finite anchoring energy for $d / p=0.25$, the lowest twist angle is chosen to be $10^{\circ}$, while the highest twist angle is $170^{\circ}$. The phase difference accumulated by the extraordinary polarized wave upon propagation through the LC cell $(50-\mu \mathrm{m}$ gap, LC E7, $d / p=0.25)$ between the areas with the high twist and low twist angles as the function of applied voltage is shown in Fig. 5.

\subsection{Design of LC lens}

The design of the LC lens based on the twist angle gradient includes two LC cells with a single electrode possessing an identical gradient of azimuthal aligning direction at one substrate (Fig. 6). Each LC cell has continuous variation of the twist angle as shown in Fig. 4. Application of the same low-voltage level to the both cells supplies the phase difference, according to Fig. 5.

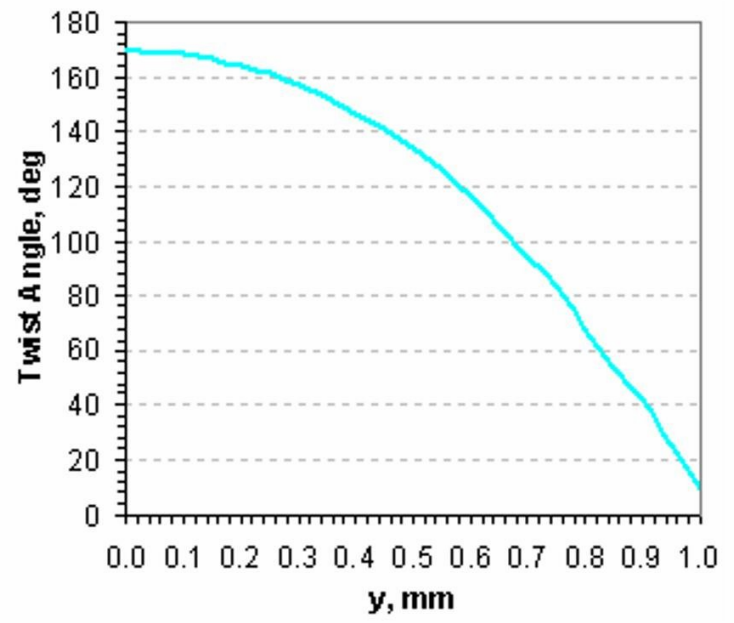

Fig. 4. Twist angle gradient required for the LC lens phase profile. 
Each LC cell is polarization dependent and affects the propagation of single polarization component. Thus, the polarization independent LC lens design has two LC cells (Fig. 6) oriented orthogonally to each other. Also, although the twist angle value is the same, the sign of the twist angle should be different to compensate polarization rotation of the twist angle gradient (Fig. 7). Thus, if the first cell is filled with LC doped with the left twisting chiral dopant, e.g. ZLI-811, Merck, then the second cell is filled with LC doped with the right twist chiral dopant, e.g. ZLI-4572, Merck.

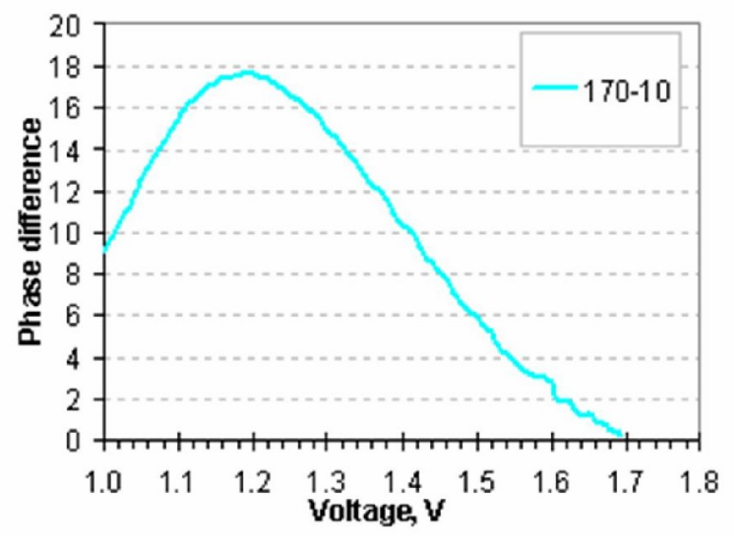

Fig. 5. Phase difference between high and low twist areas vs. the applied voltage.

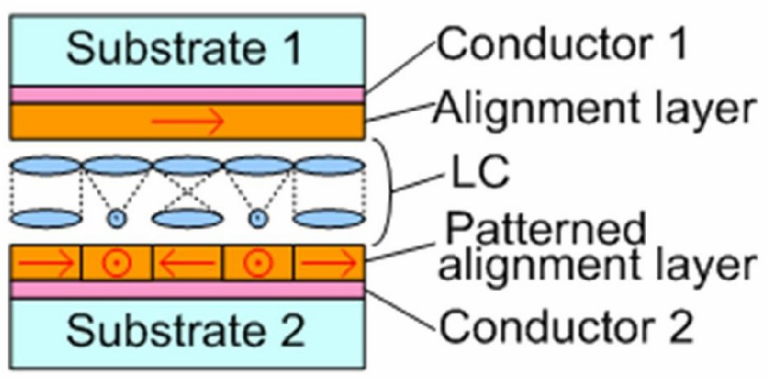

Fig. 6. Schematic structure of a single LC cell with the twist angle gradient.

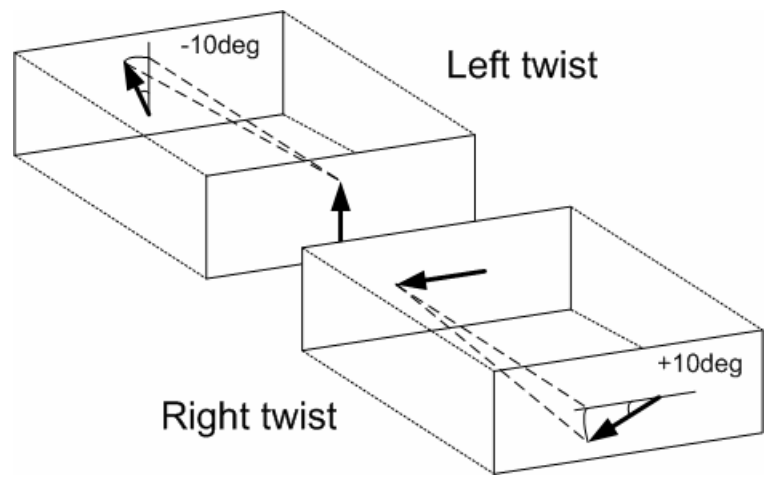

Fig. 7. Polarization independent design of the LC lens based on the twist angle gradient.

\section{Experimental verification}

The possibility to realize lens profile upon switching the LC cell with the twist angle gradient depends on the threshold voltage dependence on the twist angle inside single LC cell. To verify the dependence, we prepared LC cell with three regions of different twist angles of $45^{\circ}, 90^{\circ}$ and $135^{\circ}$ inside the cell (Fig. 8).

The cell consists of two glass substrates with ITO conductor. Uniformly rubbed Nylon6 alignment layer was prepared on the top of the substrate 1. Substrate 2 was coated with the patterned alignment layer of B-15 [8] material. The substrates were sandwiched together to form the LC cell with $20-\mu \mathrm{m}$ gap, which was filled with LC E7 doped with ZLI-811 chiral dopant. The $d / p$ ratio was 0.25 . Fig. 8 shows the photo of the LC cell with three areas of different twist angles placed between two polarizers oriented at $-45^{\circ}, 0^{\circ}$ and $45^{\circ}$. It is possible to see a dark state of the twist angle regions with $45^{\circ}, 90^{\circ}$ and $135^{\circ}$, correspondingly to each configuration of the polarizers.

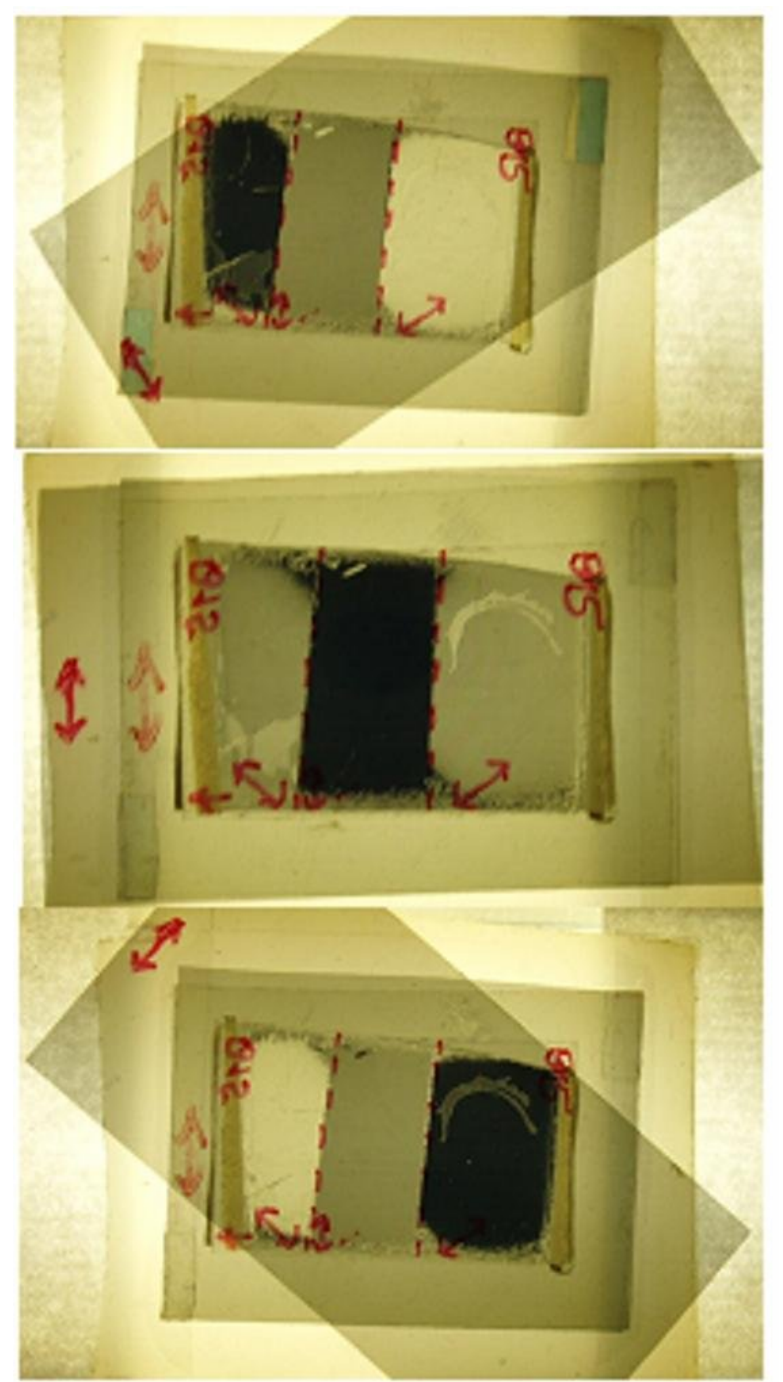

Fig. 8. Photo of the photoaligned LC cell with $45^{\circ}, 90^{\circ}$ and $135^{\circ}$ twist angle regions. 


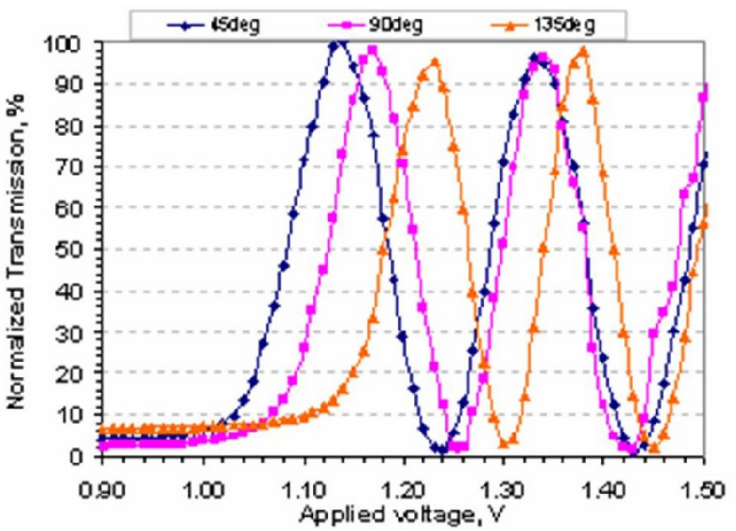

Fig. 9. Transmission-voltage characteristics of $45^{\circ}, 90^{\circ}$ and $135^{\circ}$ twist angle regions of the LC cell, angles between polarizers are $-45^{\circ}, 0^{\circ}$ and $45^{\circ}$, correspondingly.

Fig. 9 presents the transmission-voltage characteristics for different twist angle regions of the LC cell shown in Fig. 8. The angle between polarizers in each region of measurements was chosen to compensate the rotation effect of the twist structure.

As confirmed in experiment, there exists the threshold voltage dependence on the twist angle suitable for implementation of LC lens based on the gradient of the twist angle.

\section{Conclusions}

The new design of LC lens based on patterning the alignment material to create the twist angle gradient is offered. The possibility to create the LC lens based on the twist angle gradient is verified in experiment. The stack of two LC cells can be used to achieve polarization independent operation of the LC lens compensating polarization rotation of twisted LC structure. The suggested LC lens requires simple driving electronics with a single control electrode and low-voltage driving. $1.2-1.3 \mathrm{~V}$ applied to both cells simultaneously is sufficient to obtain the resultant LC lens refractive index profile of the short focal length.

\section{References}

1. M. Kawamura and S. Sato, Liquid crystal lens with divided-circularly hole-patterned electrodes // Proc. IDRC'08, P-18, p. 193-196 (2008).

2. G.D. Love and A.F. Naumov, Modal liquid crystal lenses // Liq. Cryst. Today, ISSN 1469-5181 (2000).

3. H. Ren, Y.-H. Lin, and S.-T. Wu, Adaptive lens using liquid crystal concentration redistribution // Appl. Phys. Lett. 88, 191116 (2006).

4. C.R. Lee, K.C. Lo, and T.S. Mo, Electrically switchable Fresnel lens based on a liquid crystal film with a polymer relief pattern // Jpn. J. Appl. Phys. 46, p. 4144-4147 (2007).

5. S. Valyukh, V. Chigrinov, and H.S. Kwok, A liquid crystal lens with non-uniform anchoring energy // SID Intern. Symp. Dig. Tech. Pap. 39, p. 659-662 (2008).

6. M. Ye, Y. Yokoyama, and S. Sato, Liquid crystal lens prepared utilizing patterned molecular orientations on cell walls // Appl. Phys. Lett. 89, 141112 (2006).

7. T. Scheffer and J. Nehring, In: Liquid Crystals: Application and Uses (ed. B. Bahadur), 1, p. 253. World Scientific, 1990.

8. U.V. Mahilny, A.I. Stankevich, A.A. Muravsky, A.A. Murauski, Novel polymer as liquid crystal alignment material for plastic substrates // J. Phys. D: Appl. Phys. 42, 075303 (2009). 